\title{
Evidence for selective resputtering as the growth mechanism of pair-order anisotropy in amorphous TbFe films
}

\author{
VINCENT G HARRIS* and TARAS POKHIL ${ }^{\dagger}$ \\ *US Naval Research Laboratory, Washington, DC 20375 USA \\ ${ }^{\dagger}$ Seagate Technologies, Minneapolis, MN 55455 USA
}

\begin{abstract}
Processing conditions for rf magnetron sputter-deposited amorphous TbFe films in which an atomic scale structural anisotropy (ASSA) results as a natural consequence of selective resputtering at the film surface during growth are presented. The ASSA, measured using polarization-dependent X-ray absorption fine structure with quantitative modelling via a parametrized nonlinear least squares fitting, is described as a pair order anisotropy (POA) where a statistical preference exists for like atom pairs in-plane and unlike atom pairs perpendicular to the film plane. The perpendicular magnetic anisotropy (PMA) energy increases with increasing POA for samples grown using decreasing rf power and increasing working gas pressure. The POA directly reflects the anisotropic electrostatic environment at the rare earth site which is required for PMA to result from a single ion anisotropy mechanism.
\end{abstract}

Keywords. Magnetic anisotropy; EXAFS; pair-order anisotropy; TbFe films; rf sputter deposition.

\section{Introduction}

Amorphous TbFe-based thin films typically possess perpendicular magnetic anisotropy (PMA) - a property which has proven integral to the development of magnetooptical and bubble memory technologies (Hansen and Heitmann 1989). Although PMA was first observed in amorphous rare earth-transition metal ( $a$-RE-TM) alloys in 1973 (Chaudhari et al 1973), the underlying mechanism responsible for its origin remained unclear for nearly two decades. It is widely held that a single ion anisotropy stemming from a crystal field interaction on the RE site is responsible for the large PMA. Magnetoelastic contributions from intrinsic stress and thermal expansion mismatch between film and substrate, dipole contributions from columnar morphology and free surfaces, and magnetocrystalline contributions from nanocrystals, have all been empirically ruled out as the primary source of PMA in these systems (Mizoguchi and Cargill 1979). None-the-less, some deviation from an ideal amorphous structure must exist to provide the needed anisotropic electrostatic environment for large PMA. One of the chief obstacles to solving this problem has been the inability to measure accurately and with precision the atomic structure of an amorphous sample. In the later 1980s extended X-ray absorption fine structure (EXAFS) theory and detector technology, together with advances in synchrotron radiation science, had evolved to a level which made these measurements possible.

In 1992, Harris et al (1992) reported the first polarization-dependent EXAFS measurements of $a$-TbFe films

*Author for correspondence that unambiguously showed the presence of an atomicscale structural anisotropy (ASSA). Modelling the fine structure led to a description of the anisotropy as a statistical preference for like atom pairs along the in-plane direction and unlike atom pairs perpendicular to the film plane. This description was similar to that proposed by Gambino (1973) and Gambino and Cuomo (1978) as a pair-order anisotropy (POA). The magnitude of this POA was found to scale with the measured magnetic anisotropy energy in ion-beam-sputtered thin films before and after heat treatment. In an extension of this work, Harris et al (1994) showed that the POA also scales closely with the magnetic anisotropy energy in films grown by dc magnetron sputtering as a function of deposition temperature. However, an issue that has not been satisfactorily addressed is how the POA forms in these films to give rise to the measured PMA.

The focus of this paper is three-fold: investigation of the role that processing parameters play in determining the magnetic anisotropy energy and the pair order anisotropy, and to identify the underlying growth mechanism responsible for the presence of POA.

\section{Sample processing and characterization}

A series of $a$-TbFe films, having the nominal composition $\mathrm{Tb}_{18.6} \mathrm{Fe}_{21.4}$, were processed using if magnetron sputterdeposition. The rf power and Ar gas pressure varied from $250-1000 \mathrm{~W}$ and $3.8-7.5 \mathrm{mT}$ respectively, resuiting in a systematic change in magnetic anisotropy energy (see table 1). The films were grown to thickness of $740-920 \AA$ on glass substrates with a $200 \AA \mathrm{Si}_{3} \mathrm{~N}_{4}$ under 
and overlayer. The rate of deposition was dictated by the processing parameters and ranged from $100-370 \AA / \mathrm{min}$.

The static magnetic properties of the samples were determined using an alternating gradient magnetometer which provided $M$ vs $H$ curves. The uniaxial magnetic anisotropy energy was determined using the relationship $K_{\mathrm{u}}=2 M H_{\mathrm{a}}$ and corrected for demagnetization energy. The anisotropy field values were determined by extrapolation of the linear portion of the susceptibility to the saturation limit (Chikazumi and Charap 1964). This approach was found to produce nearly identical results as the single point method (Asti and Rinaldi 1972).

Structural characterization was based on polarizationdependent extended X-ray absorption fine structure measurements and analysis. Extended X-ray absorption spectra were collected using a total electron yield technique at the Naval Research Laboratory's Materials Analysis beamline (X23B) at the National Synchrotron Light Source (Brookhaven National Laboratory, Upton, NY). Harris and Elam (1997) described this detection scheme and the performance characteristics of the detector used here. Data was collected using normal and glancing angle $\left(10^{\circ}\right.$ with respect to the film plane) incident radiation. Because synchrotron radiation is linearly polarized and the EXAFS signal experiences a $\cos ^{2}$-dependence with respect to the electric field vector of the incident radiation, these orientations allow the sampling of in-plane and out-of-plane structure, respectively. EXAFS analysis and modelling procedures used here follow those outlined by Sayers and Bunker (1988). In the modelling analysis, theoretical EXAFS data was generated using the FEFF codes of Rehr et al (1992) and refined by fitting to pure phase standard of $\mathrm{TbFe}_{2}$.

\section{Selective resputtering}

When an energetic ion is incident upon a target material, as is the case in all sputter-deposition techniques, including if magnetron sputtering, one or more of five events may occur: (i) the incident ion can impact the target and produce secondary electrons; (ii) the ion may be buried within the surface of the target (i.e. implantation); (iii) the impact may cause structural rearrangements at the target surface (i.e. surface damage); (iv)

Table 1. Processing conditions and magnetic anisotropy energies.

\begin{tabular}{lcccc}
\hline $\begin{array}{l}\text { Sample } \\
\mathrm{Tb}_{19} \mathrm{Fe}_{81}\end{array}$ & $\begin{array}{c}\text { Ar pressure } \\
(\mathrm{mT})\end{array}$ & $\begin{array}{c}\text { rf power } \\
(\mathrm{W})\end{array}$ & $\begin{array}{c}\text { Thickness } \\
(\AA)\end{array}$ & $\begin{array}{c}E_{\mathrm{a}} \\
(\mathrm{ergs} / \mathrm{cc})\end{array}$ \\
\hline I & 7.5 & 250 & 790 & $1.3 \times 10^{6}$ \\
II & 7.5 & 500 & 825 & $6 \times 10^{5}$ \\
III & 7.5 & 1000 & 740 & $2 \times 10^{5}$ \\
IV & 3.8 & 500 & 920 & $5 \times 10^{4}$ \\
\hline
\end{tabular}

the ion may be reflected from the target surface retaining its kinetic energy; and/or (v) the ion may impact the target, experience one or more elastic collisions and cause the ejection of one or more neutral atoms from the surface (i.e. sputtering) (Chapman 1980). Alternatively, the substrate is also bathed in a sea of particles of varying charge and energy, which interact with the film during growth. These particles include: sputtered atoms, photons, negative ions, fast and slow electrons, thermal neutrals, fast metastables resulting from charge transfer (e.g. $\mathrm{Ar}^{++}, \mathrm{Ar}^{+}, \mathrm{Ar}^{+2}$ ) and target-reflected neutrals (Ar), as well as contaminants and ionized complexes (e.g. $\mathrm{ArH}^{+}$and $\mathrm{H}_{3} \mathrm{O}^{+}$). Compared with all other particles incident upon the substrate, the flux of Ar atoms and ions is clearly dominant (Chapman 1980).

In order to explore effect of the flux of $\mathrm{Ar}$ ions and neutrals have on the growing film, one can examine the likely adatom arrangements at the film surface and estimate the binding energies for each. From this, the likelihood of an adatom being re-sputtered from the film surface can be calculated. Because each type adatom arrangement will have a different binding energy, the threshold energy for resputtering is also different for each arrangement. This leads to the possibility that one adatom is more likely to be re-sputtered from the film surface than another (i.e. selective resputtering), and may lead to deviations from target stoichiometry of the resulting film, or a more subtle effect the creation of anisotropic pair correlations, i.e. a statistical preference for the spatio-anisotropic distribution of like atom pairs (a.k.a pair-order anisotropy) (Gambino and Cuomo 1978).

Table 2 lists the likely adatom arrangements for the case of $\mathrm{TbFe}$ films studied here, with their estimated binding energies and threshold energies. Adatom arrangements that have low probabilities of occurring, owing to film composition, are not shown. The binding energies were estimated to be equal to the values applied by Gambino and Cuomo (1978) for the case of GdCo. Since the ion energies in an rf plasma do not typically exceed a couple of hundred eV (Toups and Ernie 1990), the ion collisions are those of the single knockon regime (Chapman 1980). In the simplest case, the incident ion experiences two elastic collisions. The first is a collision with an atom within the lattice where the ion is backscattered towards the film surface. The second collision is with a surface adatom which results in the sputtering of that atom from the film surface. The energies needed by the $\mathrm{Ar}$ ion (i.e. threshold energies) to communicate sufficient energy to the adatom for it to overcome the surface work function are determined for the case where the first and second collisions are with $\mathrm{Fe}$ and $\mathrm{Tb}$ atoms. If the Ar ion incident at the film surface has $E>\sim 65 \mathrm{eV}$ then re-sputtering takes place without a selective character. Correspondingly, if the $\mathrm{Ar}$ ions have $E<\sim 34 \mathrm{eV}$, the energy communicated via an elastic 
collision to the adatom is insufficient to overcome the surface work function and no resputtering takes place. However, if the energy falls between these values then some adatoms are selectively resputtered resulting in the above mentioned POA.

One difficulty in determining the probability of selective resputtering is knowing the energy distribution of the Ar ions incident upon the substrate film. In this case, we have benefited greatly from the work of Toups and Ernie (1990). In their work, they experimentally determined the energy distribution and flux of Ar ions bombarding an electrode in a parallel plate rf reactor. Their experiment bears resemblance to the geometry and conditions used in the rf magnetron sputter deposition of the films studied by us, and their results are extendable to our processing studies. Figure 1 is a plot of the ion energy vs working gas pressure as a function of $\mathrm{rf}$ power. The solid symbols are derived from the work of Toups and Ernie (1990), while the open symbols represent the films of the present study. The solid horizontal bar represents the position of the maximum of the ion energy distribution which is expected to produce the most selective resputtering events and lead to the largest POA. The distribution of ion energies has a sharp cutoff at high energies and a long tail at low energies. Therefore, the maximum of the distribution is shifted towards the upper limit where most of the plasma ions will satisfy the selective resputtering conditions.

\section{Atomic-scale structural anisotropy}

In order to measure the POA, believed to be present in these film samples, we have applied PD-EXAFS, which provides element-specific directional-sensitive atomic structure information. The $\mathrm{X}$-ray absorption coefficient was measured over the range of both $\mathrm{Fe} K$ and $\mathrm{Tb} \mathrm{L}_{\mathrm{III}}$ absorption edges on each the films listed in table 1

Table 2. Adatom arrangements, binding energies and threshold energies for selective resputtering.

\begin{tabular}{ccc}
\hline $\begin{array}{l}\text { Adatom } \\
\text { arrangement }\end{array}$ & $\begin{array}{c}\text { Binding energy } \\
(\mathrm{eV})\end{array}$ & $\begin{array}{c}\text { Threshold energy } \\
(\mathrm{eV})\end{array}$ \\
\hline 0 & 13 & 56 \\
8 & 15 & 65 \\
8 & 14 & 40 \\
8 & 13 & 37 \\
\hline
\end{tabular}

using both normal and glancing incident photons to measure the in-plane and perpendicular components of element-specific local structure, respectively. This technique was employed by Harris et al $(1992,1994)$ to show the existence of a structural anisotropy between the in-plane and out-of-plane structure in $a$-TbFe films.

Figure 2 shows Fourier transformed Fe EXAFS data collected using normal (solid) and glancing (dashed) incident radiation. Electron phase shifts have not been corrected for at this stage in the analysis and therefore the Fourier transform peak positions do not reflect true radial distances but are instead shifted to lower $r$ values. However, comparisons between transforms are effective in determining relative changes in the local structure around the absorbing atom. The data in figures 2 (a)-(d) corresponds to samples I-IV of table 1, respectively. The anisotropy which exists between data sets is indicated by the circle in figure $2 \mathrm{a}$, and is manifested primarily in the near neighbour peak centred near $2 \AA$. This peak corresponds to the near neighbour coordination sphere around the $\mathrm{Fe}$ atoms and includes contributions from $\sim 10 \mathrm{Fe}$ nearest neighbours and $1-2 \mathrm{~Tb}$ nearest neighbours as dictated by the film composition. Table 1 shows the magnetic anisotropy energy to scale as $K_{\mathrm{u}(\mathrm{I})}>K_{\mathrm{u}(\mathrm{II})}$ $>K_{\mathrm{u}(\mathrm{IV})}>K_{\mathrm{u}(\mathrm{III})}$. This is depicted graphically in figure 3 where $K_{\mathrm{u}}$ is plotted against both rf power and working gas pressure. An inspection of the NN regions of figures $2 a-d$ indicates that the difference between data sets scales with the measured magnetic anisotropy energy values.

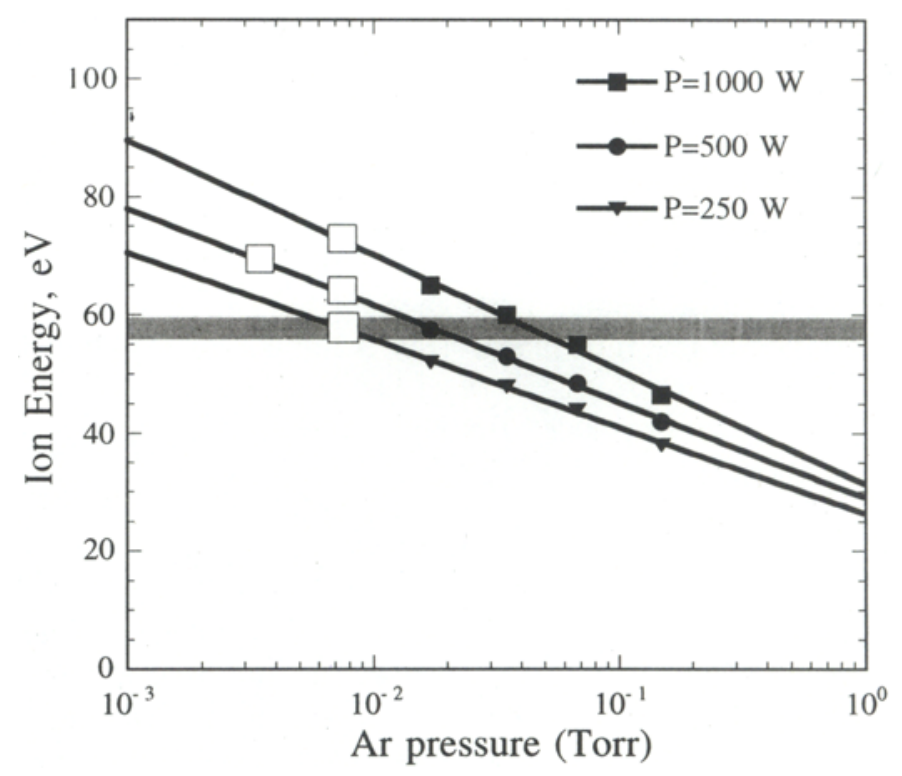

Figure 1. Ion energies as a function of working gas pressure and $\mathrm{rf}$ power. The horizontal bar indicates the energy at which selective resputtering is maximized. The solid symbols are derived from Toupe and Ernie (1990). The open symbols correspond to the samples of table 1 . 


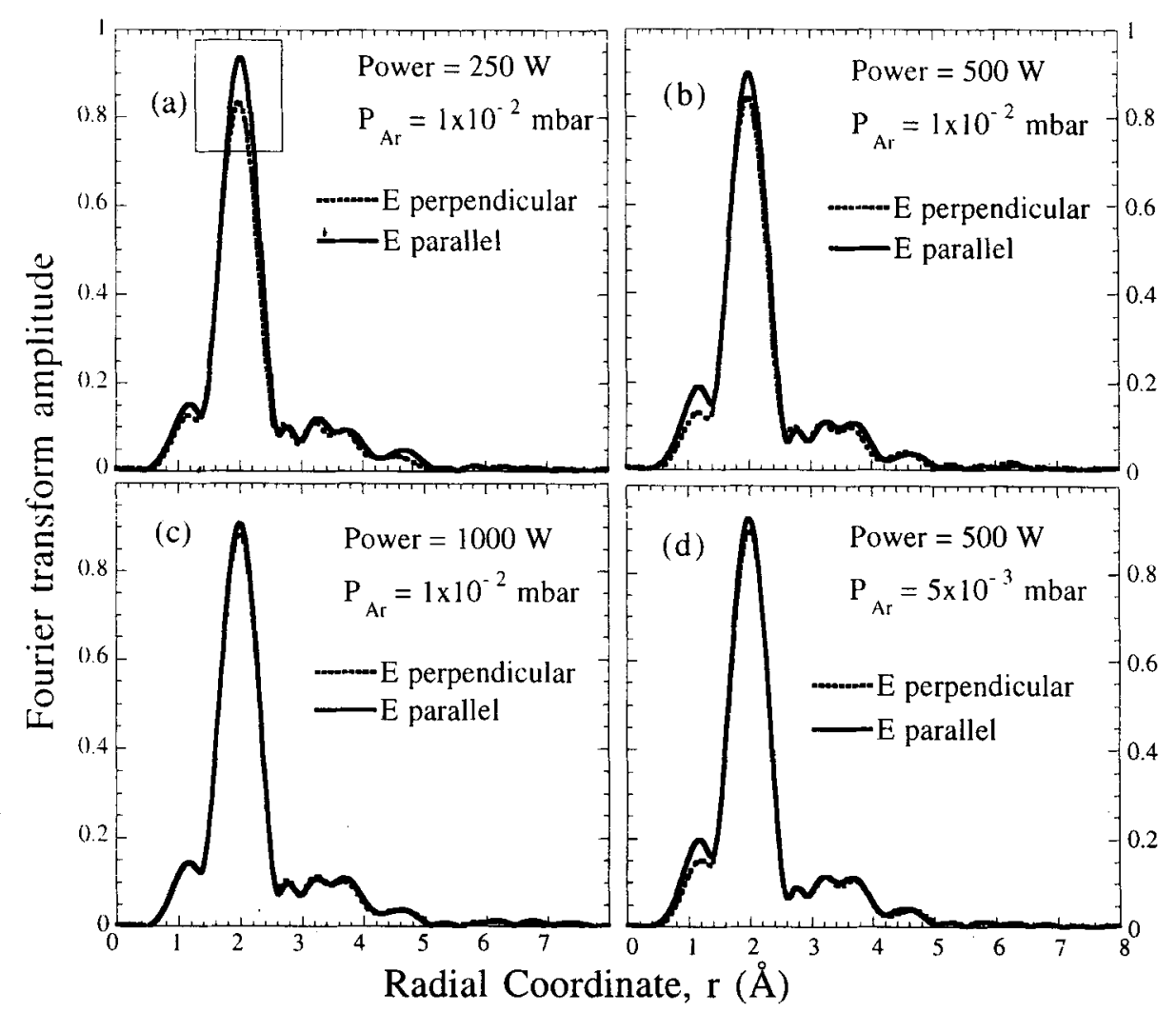

Figure 2. Fourier transformed Fe EXAFS data for the samples of table 1 (figure $\mathbf{2 a}, \mathbf{b}$, c and d, respectively) using both normal and incident angle radiation. The atomic scale structure anisotropy (ASSA) is most evident in the near neighbour amplitude (see circle in a). A k range of $2-10 \AA^{-1}$ with a $\mathrm{k}^{2}$ - weighting was applied during the Fourier transformation.

Applying a parametrized nonlinear least squares fitting approach (Stern 1992), we have determined that the anisotropy seen in figure 2 is the result of a statistical preference for like neighbour pairs in the film plane with a corresponding preference for unlike pairs perpendicular to the film plane. This is similar to that found previously by Harris et al $(1992,1994)$ and consistent with the POA model first proposed by Gambino and Cuomo (1978).

\section{Correlation of $K_{u}$ with POA}

Figure 3 depicts the dependence of $K_{\mathrm{u}}$ on the if power and working gas pressure used in processing. With increasing If power, $K_{u}$ decreases in a continuous fashion. Correspondingly, with increasing gas pressure, $K_{\mathrm{u}}$ increases and then rolls over and decreases at a pressure of $\sim 11 \mathrm{mT}$. This roll over effect is likely due to the increase in thermalizing collisions experienced by the high energy atoms on their way to the substrate. In the latter curve, our data points are plotted along with the data of Kavalerov et al (1993), who has explored samples over a broader pressure range. From figure 1 it can be seen that increasing power, with all other processing parameters fixed, moves the $\mathrm{Ar}$ ion energy away from the condition of maximum selective resputtering. Further, increasing working gas pressure leads to lower ion energies which favour the selective resputtering of $\mathrm{Fe}$ adatoms over $\mathrm{Tb}$ adatoms. Both of these trends in Ar ion energy correlate closely and with the proper sign for the dependence of $K_{u}$ and can be reconciled using only the effects of selective resputtering.

In figure 4 the measured $K_{\mathrm{u}}$ is plotted against a POA metric determined from PD-EXAFS. The metric is per cent change in $\mathrm{Fe}-\mathrm{Fe}$ atom pairs between the in-plane and out-of-plane structures. As can be seen, there is a strong positive correlation between the $K_{\mathrm{u}}$ and POA, namely that the $K_{\mathrm{u}}$ values increase linearly on a $\log$ scale with respect to the POA. This type of relationship is also seen in dc magnetron-sputtered films grown at 


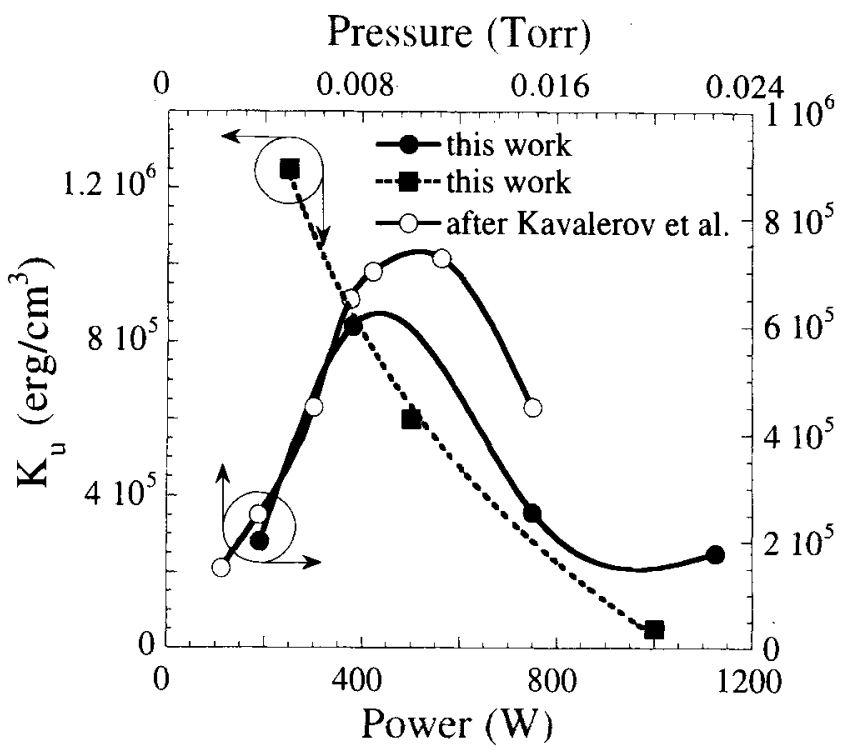

Figure 3. Magnetic anisotropy constant $K_{u}$ as a function of rf power and working gas pressure used in processing.

different deposition temperatures (Harris et al 1994) and ion-beam-sputtered films before and after heat treatment (Harris et al 1992). Because all films are presumed to be in the state of tension, as shown by the work of Thornton and Hoffman (1989), and that the magnetostrictive coefficient is positive for amorphous TbFe (Clark 1980), one does not expect magnetoelastic interactions to contribute to the PMA, but instead lesson the effect. For the samples having the smaller $K_{u}$ values, and correspondingly none or little POA, one must speculate as to the source of the anisotropy. Residual POA, that is beyond the detection limits of our technique, or dipole contributions from a columnar morphology, are two mechanisms that may account for the $K_{\mathrm{u}}$ values for these samples.

The underlying physical mechanism of the PMA in $a$-RETM systems is certainly a single ion anisotropy resulting from a crystal field interaction on the RE site. In order for this mechanism to provide large PMA, the local electrostatic environment must contain a strong anisotropy with respect to the film plane. The POA measured here and in previous studies provides a direct measure of the anisotropic chemical environment that results in an anisotropic electrostatic environment on the RE site. The strong correlation between increasing POA and increasing $K_{\mathrm{u}}$ supports this interpretation. Further, on examining the source of PMA in RETM intermetallics, it can be observed that the magnetic anisotropy is largest along the crystal directions having the greater number, unlike near-neighbour pairs (Coehoorn 1991). This is consistent with the results of this study which too shows that the PMA resides along the direction of unlike pairings.

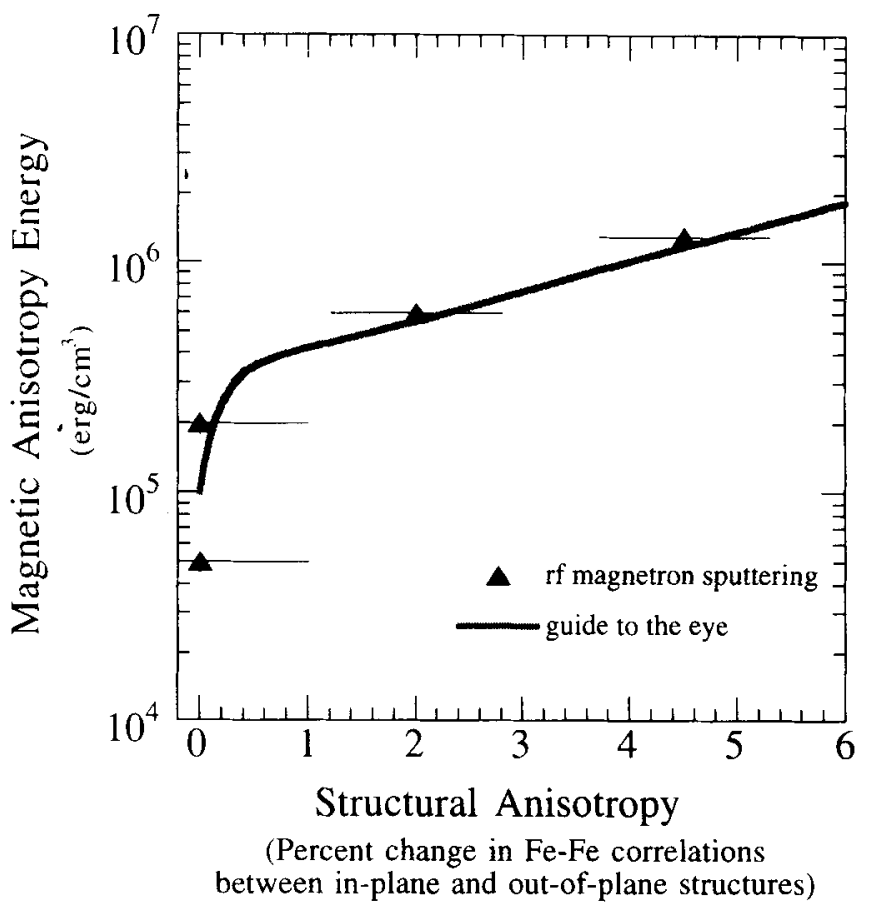

Figure 4. Magnetic anisotropy constant $K_{\mathrm{u}}$ plotted against POA metric, i.e. per cent change in the $\mathrm{Fe}-\mathrm{Fe}$ pairs between the in-plane and perpendicular directions.

\section{Conclusions}

We have defined processing conditions in the $\mathrm{rf}$ magnetron sputter-deposition of $a$-TbFe films in which $\mathrm{Ar}$ ion possess energies that lead to selective resputtering at the film surface during growth. $K_{\mathrm{u}}$ has been observed to increase with working gas pressure and decreasing rf power in rf magnetron-sputtered amorphous $\mathrm{TbFe}$ films. Using PD-EXAFS, we have measured an atomic-scale structural anisotropy (ASSA) between the in-plane and out-of-plane direction of the films. This ASSA is described using a parametrized least squares fitting of the EXAFS as a statistical preference for like atom pairs along the in-plane direction and unlike atom pairs perpendicular to the film plane. We define this local atomic arrangement as a pair-order anisotropy (POA) after Gambino and Cuomo (1978). $K_{\mathrm{u}}$ and the measured POA have been found to have a strong positive correlation, where the $\log$ of $K_{\mathrm{u}}$ increases linearly with the measured POA metric (per cent change in the $\mathrm{Fe}-\mathrm{Fe}$ pairs between the in-plane and perpendicular directions). We have shown that the POA scales with $K_{\mathrm{u}}$ as a function of Ar ion energy that promotes the selective resputtering of $\mathrm{Fe}$ atoms bonded in part to subsurface $\mathrm{Tb}$ atoms, leading to the observed POA. This POA directly reflects the anisotropic electrostatic environment at the rare earth site which is required for PMA to result from a single-ion anisotropy mechanism. 


\section{References}

Asti G and Rinaldi S 1972 Phys. Rev. Lett. 281584

Chapman B 1980 Glow discharge processes: sputtering and plasma etching (New York: John Wiley \& Sons, Inc.)

Chaudhari P, Cuomo J J and Gambino R J 1973 Appl. Phys. Lett. 22337

Chikazumi S and Charap S H 1964 Physics of magnetism (New York: John Wiley \& Sons, Inc.)

Clark A E 1980 Magnetostrictive rare earth $\mathrm{Fe}_{2}$ compounds; in Ferromagnetic Materials (ed.) E P Wohlfarth (Amsterdam: North Holland Publishing Co) 1 pp. 531-589

Coehoom R 1991 J. Magn. Magn. Mater. 9955

Gambino R J and Cuomo J J 1978 J. Vac. Sci. Tech. 15296

Gambino R J et al 1973 AIP conf. proc. 18 578-592

Hansen P and Heitmann H 1989 IEEE Trans. on Magn. 25 4390
Harris V G et al 1992 IEEE Trans. on Magn. 282955

Harris V G et al 1992 Phys. Rev. Lett. 691939

Harris V G and Elam W T 1997 Rev. Sci. Inst. 681972

Harris V G et al 1994 Phys. Rev. B49 3637

Kavalerov V G, Vredensky B S and Kochetkov V V 1993 IEEE Trans. on Magn. 293111

Mizoguchi T and Cargill G S III 1979 J. Appl. Phys. 50 3570

Rehr J J, Zabinsky S I and Albers R C 1992 Phys. Rev. Lett. 693397

Sayers D E and Bunker B A 1988 in X-ray absorption: Principles applications techniques of EXAFS, SEXAFS and XANES (eds) D C Koningsberger and R Prins (New York: John Wiley \& Sons) 92 pp. 211-253

Stern E A 1992 Phys. Rev. B46 687

Thornton J A and Hoffman D W 1989 Thin Solid Films 171 5

Toups M F and Emie D W 1990 J. Appl. Phys. 686125 